2. That step adjustments shall be exactly $1^{\mathrm{s}}$. When a step adjustment is made it shall be at $0^{\mathrm{h}}$ on the first day of a month with preference for 1 January or 1 July. These step adjustments will be decided upon and announced as early as possible by the BIH.

3. The maximum difference UT1-UTC will be less than 0.7 unless there are exceptional variations in the rotation of the Earth.

4. Special adjustment. The BIH will also announce a unique fraction of a second adjustment to be made at $0^{\text {h }} 1$ January 1972, so that UTC and the International Atomic Time Scale (IAT, in French TAI) will differ by an integral number of seconds.

5. The emission times of time signals from co-ordinated stations shall be kept as close to UTC (BIH) as feasible with a maximum tolerance of $1 \mathrm{~ms}$.

6. Nomenclature

6.1. Clocks in common use will indicate the minutes, seconds and fractions of UTC (French: TUC).

6.2. The terms 'G.M.T.' and ' $Z$ ' are accepted as the general equivalents of UTC in navigation and communications.

7. The term $\Delta \mathrm{UT}$ is defined by: $\Delta \mathrm{UT}=\mathrm{UT1}$-UTC. Extrapolated and final values of $\Delta \mathrm{UT}$ will be issued by astronomical observatories and the $\mathrm{BIH}$, and will be given the widest possible distribution.

8. All standard time signal emissions must include information which will enable a user to obtain UT1 with a precision of at least 0.1 .

9. Designation of the epoch of steps in UTC

9.1. If UTC is to be advanced, then second 00 will follow $23^{\mathrm{h}} 59^{\mathrm{m}} 58^{\mathrm{s}}$ of the previous day.

9.2. If UTC is to be retarded, then the second of the previous day $23^{\mathrm{h}} 59^{\mathrm{m}} 58^{\mathrm{s}}$ will be followed by the next second $0^{\mathrm{h}} 00^{\mathrm{m}} 00^{\mathrm{s}}$ of the first day of the month.

9.3. The stepped second will be commonly referred to as a "leap" second (in French: intercalaire).

9.4. The time of an event given in the old scale, before the leap second, will be given as a date in the previous month, exceeding $24^{\mathrm{h}}$ if necessary. The time of an event given in the scale after the step will be given as a date in the new month, with a negative time, if necessary.

Note: Commission 31, taking into account the conflicting requirements of the various users of UTC, including the large number of those requiring immediate knowledge of hour angle, considers that the above represents the optimum solution.

\title{
JOINT MEETING OF COMMISSIONS 4 AND 31, ON TIME SCALES, 25 AUGUST 1970
}

Chairman: G. A. Wilkins.

Secretaries: C. J. A. Penny and A. T. Sinclair.

UNIVERSAL TIME

G. A. Wilkins drew the attention of members of Commission 31 to the resolution of D. H. Sadler (see pp. 60-63) which had been approved at the previous meeting of Commission 4 . This resolution requested that adequate means should be provided for making the difference UT1-UTC available to a precision of 0.1 before UTC is permitted to depart from UT 1 by more than about 0.1 . W. Markowitz asked why a precision of 0.1 was necessary since he doubted whether it was possible to determine positions to the corresponding accuracy of about $100 \mathrm{~m}$. In reply, R. L. Duncombe said that observational errors by navigators were unavoidable, but the time errors should be kept below the level where they would contribute to the result. R. F. Haupt said that the almanacs were designed to allow the determination of positions to $0.1 \mathrm{~min}$ of arc, and for this a precision in time of $0.25 \mathrm{sec}$ was required.

The meeting then discussed Resolution No. 1 of Commission 31 (see p. 123). R. L. Duncombe 
asked why the maximum permissible difference between UTC and UTl had been fixed at 0.7 , instead of 0.5 . G. M. R. Winkler replied that it was expected that a maximum tolerance of 0.7 could be adhered to by making step changes on the first day of a month only, whereas a maximum tolerance of 0.5 could necessitate changes on any day at short notice. After further discussion the resolution was approved by the meeting.

H. M. Smith asked for suggestions as to the best way of transmitting in coded form the corrections to UTC to obtain UTl, and if any greater accuracy than 0.1 was required in these corrections. G. E. Taylor thought that the corrections should not be incorporated in the time signal emissions, as those who needed to know the corrections immediately were frequently working in conditions of poor radio reception. Also observers who used the time signals by ear could be upset by a variation of the rythmic beat of the signals due to the coded corrections. He agreed with an idea that had been suggested of broadcasting the corrections with the shipping forecast. $\mathrm{H}$. Enslin had had experience of using a coding system, consisting of an extra signal either shortly before or shortly after the minute signal. He had found it easy to use in practice. A. M. Sinzi commented that in this sort of system signals denotating a negative correction which come just before the minute signal are easy to miss. G. A. Wilkins pointed out that the navigator would only have to note the correction once a month, as the resolution proposes that step changes should only be made on the first day of a month. Everyone was agreed that no greater precision than 0.1 was needed in the correction UT1-UTC at this time.

\section{EPHEMERIS TIME}

G. A. Wilkins stated that a working group on units and time scales had been set up by Commission 4, with himself as convener. Part of its work would be to look into the possible need for a new definition of the ephemeris time scale.

T. C. Van Flandern gave a summary of his paper entitled 'The need for a new ephemeris time scale', in which he discussed evidence that the present Ephemeris Time scale is not supported by observations of the Sun, Moon, or inner planets. The present Ephemeris Time scale depends on the adopted values for certain parameters, such as the equinox location and the tidal acceleration of the Moon. In the light of modern revisions of the values of these parameters he suggested several possibilities for the revision of the definition of Ephemeris Time.

B. Guinot thought that the working group set up by Commission 4 should consider using Atomic Time in ephemerides, so that Ephemeris Time would no longer be required. T. C. Van Flandern agreed with this for future ephemerides, but said that the best way to extend a time scale backwards was by using the Moon to give Ephemeris Time. I. I. Shapiro said that the variation of the time scale used in the past could be taken as an unknown to be determined by the comparison of theories with observations. A. Stoyko presented figures for the variation of Ephemeris Time from Atomic Time apparently contradictory to those of T. C. Van Flandern, but it was agreed that the two sets of figures were not comparable.

\section{RELATIVISTIC EFFECTS ON TIME SCALES}

G. Becker gave a short description of the nature of time in relativity theory. He said that time scales vary with the gravitational field, so to define a time scale it is necessary to specify the gravitational field. On the Earth we should take the gravitational field on the geoid surface as the reference field, and the clocks used to determine the time scales must be at rest. Such a time scale would be coordinate time. The variations in time scales due to changes in the gravitational potential are smaller than the errors in the best clocks available today, and so he suggested that there was no need at present to talk about coordinate time.

I. I. Shapiro attempted to clarify the difference between Ephemeris Time and coordinate time. He said that Ephemeris Time only had a meaning in Newtonian theory, and the coordinate time of relativity theory could not be compared with it. G. M. R. Winkler said that what was required was a simple system for practical time keeping on the Earth's surface. 